ORIGINAL ARTICLE

\title{
Deficient $\alpha$ smooth muscle actin expression as a cause of intestinal pseudo-obstruction: fact or fiction?
}

\author{
E Gamba, N J Carr, A C Bateman
}

J Clin Pathol 2004;57:1 168-1171. doi: 10.1136/jcp.2003.015297

See end of article for authors' affiliations .....................

Correspondence to: Dr A C Bateman, Department of Cellular Pathology, MPO02, Level E, South Block, Southampton General Hospital, Tremona Road, Southampton, Hampshire S016 6YD; adrian. bateman@suht.swest.co.uk

Accepted for publication 4 April 2004
Aims: To test the hypothesis that deficient $\alpha$ smooth muscle actin (ASMA) expression in intestinal smooth muscle, as assessed by immunohistochemistry, is specifically associated with clinical evidence of intestinal pseudo-obstruction.

Methods: Seventeen archival, formalin fixed, paraffin wax embedded samples of small intestine and 12 samples of large intestine were studied. Two of the small bowel samples and one large bowel sample were from patients with symptoms of intestinal pseudo-obstruction. The controls were longitudinal surgical margins from hemicolectomies performed for carcinoma. Immunohistochemistry was performed using primary antibodies to ASMA, smooth muscle myosin heavy chain (SMMHC), and desmin. The relative intensities of immunohistochemical expression in the circular and longitudinal muscle layers of the muscularis propria were assessed in each sample, for all three markers.

Results: All samples showed strong SMMHC and desmin expression in the inner circular and outer longitudinal layers of the muscularis propria. Both small intestinal samples from the cases and 11 of 15 controls showed no or minimal ASMA expression in the inner circular layer, with the remaining four controls also showing ASMA labelling in this layer that was weaker than within the longitudinal muscle. In contrast, intense ASMA expression was seen in both muscle layers within the large intestine in the remaining case, and in the controls.

Conclusions: There is insufficient evidence from this study to support the hypothesis that ASMA deficiency in intestinal smooth muscle, as determined by immunohistochemistry on archival tissues, is specifically associated with intestinal pseudo-obstruction.
$\mathrm{T}$ he normal motility of the gastrointestinal tract relies on coordinated neuromuscular function, the loss of which results in abnormal bowel transit as a result of altered motility. ${ }^{1}$ Six distinct actin filament isoforms have been described, of which the best known is $\alpha$ smooth muscle actin (ASMA). Actins of various isoforms are widely expressed but are not always involved in cellular contraction. ${ }^{2}$ In contrast, mature intestinal smooth muscle expresses mainly ASMA and $\gamma$ smooth muscle actin. ${ }^{3}$

The term "chronic intestinal pseudo-obstruction" refers to a clinical syndrome of recurrent intestinal obstruction caused by nerve or muscle dysfunction. ${ }^{1}$ Myopathies may affect the intestine in a segmental, multifocal, or diffuse manner. ${ }^{4}$ Malnutrition may result from untreated disease. ${ }^{5}$

\section{"The term chronic intestinal pseudo-obstruction refers to a clinical syndrome of recurrent intestinal obstruction caused by nerve or muscle dysfunction"}

In many cases of intestinal pseudo-obstruction, abnormalities may not be visible in routine haematoxylin and eosin stained sections, and additional histochemical, immunohistochemical, and ultrastructural examinations may be needed for a more precise diagnosis. In 1992, Smith et al described the case of a woman who had experienced chronic constipation since early childhood, and who had intestinal pseudoobstruction associated with the absence, as detected using immunostaining on frozen tissue, of ASMA within the intestinal circular muscle, with no abnormalities in other contractile proteins or morphological changes identified by light microscopy or ultrastructural examination. ${ }^{6}$ This finding has been subsequently classified as a cause of primary congenital/early onset intestinal myopathy, as a primary specific contractile protein abnormality in apparently normal smooth muscle. ${ }^{3}$

In our study, we have tested the hypothesis that chronic intestinal pseudo-obstruction is specifically associated with deficient ASMA expression within the inner circular layer of the intestinal muscularis propria, as assessed using immunohistochemistry on formalin fixed and paraffin wax embedded material.

\section{METHODS}

Three patients with intestinal pseudo-obstruction were studied. In two patients, the small intestinal wall was available and in the third case, a sample of the large intestine was studied. Case 1 was a 41 year old woman with an eight year history of abdominal pain, in whom small bowel dysmotility had been diagnosed after manometry studies, and who had previously undergone right hemicolectomy for a caecal volvulus. Case 2 was a 28 year old woman with a clinical diagnosis of small intestinal dysmotility, who underwent laparoscopy and full thickness ileal biopsy. Case 3 was a 54 year old woman with a history of chronic constipation since adolescence and a clinical diagnosis of pseudo-obstruction who underwent subtotal colectomy. No mechanical cause for bowel obstruction was identified within the resection specimens.

Control material was sourced from archival resections from 15 patients undergoing right hemicolectomy for caecal or proximal colonic carcinoma. Once suboptimally orientated sections had been excluded, a total of 15 small intestine samples and 11 large intestine samples served as controls.

Abbreviations: ASMA, $\alpha$ smooth muscle actin; SMMHC, smooth muscle myosin heary chain 

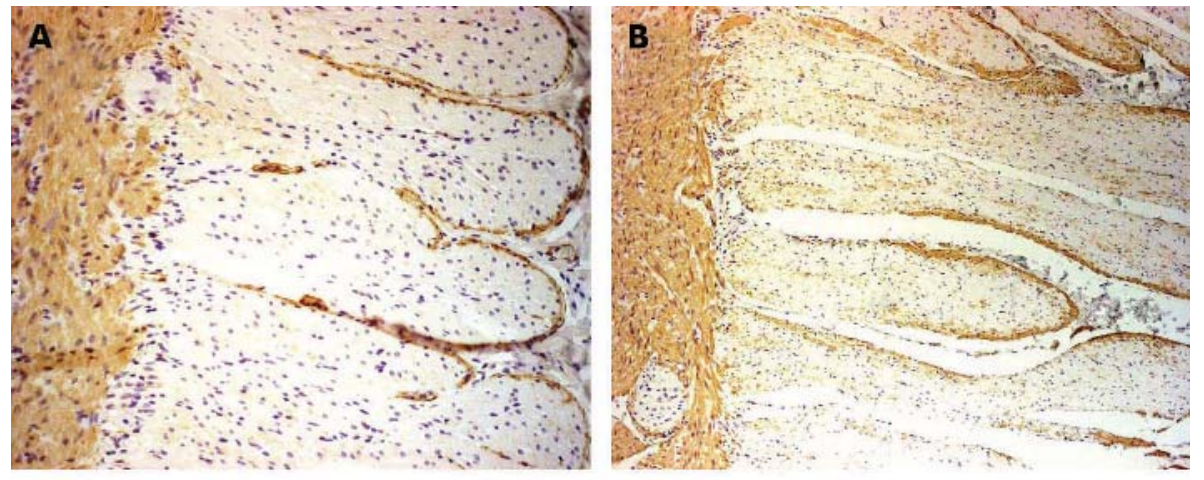

Figure 1 (A-D) Immunohistochemical staining for $\alpha$ smooth muscle actin. (A) Case 1 (small intestine) and (B) control 1 (small intestine), both demonstrating absent expression within the bulk of the circular muscle and expression within the longitudinal muscle and the innermost layer of circular muscle. (C) Control 13 (small intestine) showing weak expression within the circular muscle. (D) Case 3 (large intestine) showing equally intense expression within both the circular and longitudinal muscle. (E, F) Immunohistochemical staining for desmin. (E) Case 1 (small intestine) and (F) control 1 (small
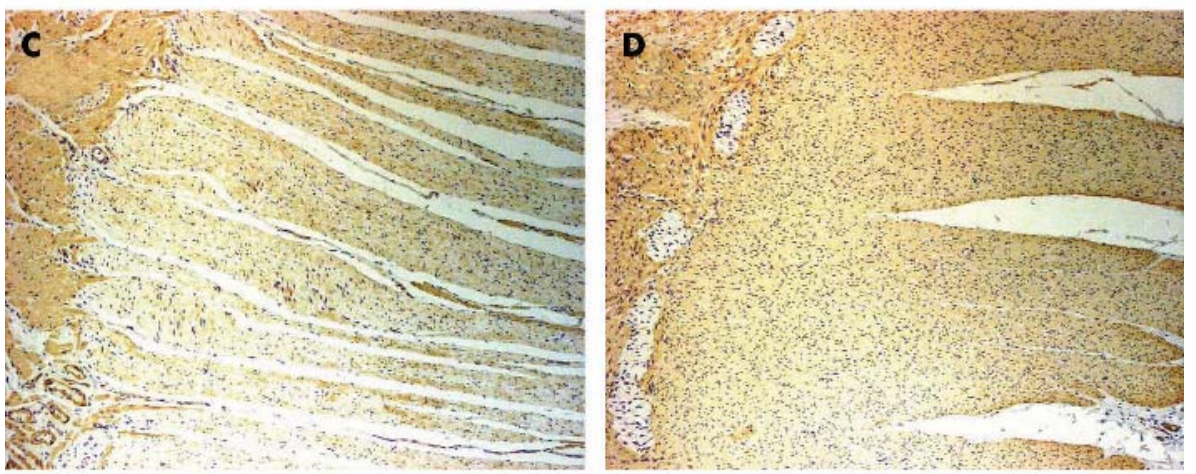
intestine), both demonstrating equally intense expression within the circular and longitudinal muscle. $(G, H)$ Immunohistochemical staining for smooth muscle myosin. (G) Case 1 (small intestine) and (H) control 1 (small intestine), both demonstrating equally intense expression within the circular and longitudinal muscle. All of the figures are orientated such that the outer longitudinal muscle is on the left side and the inner circular muscle is on the right side.
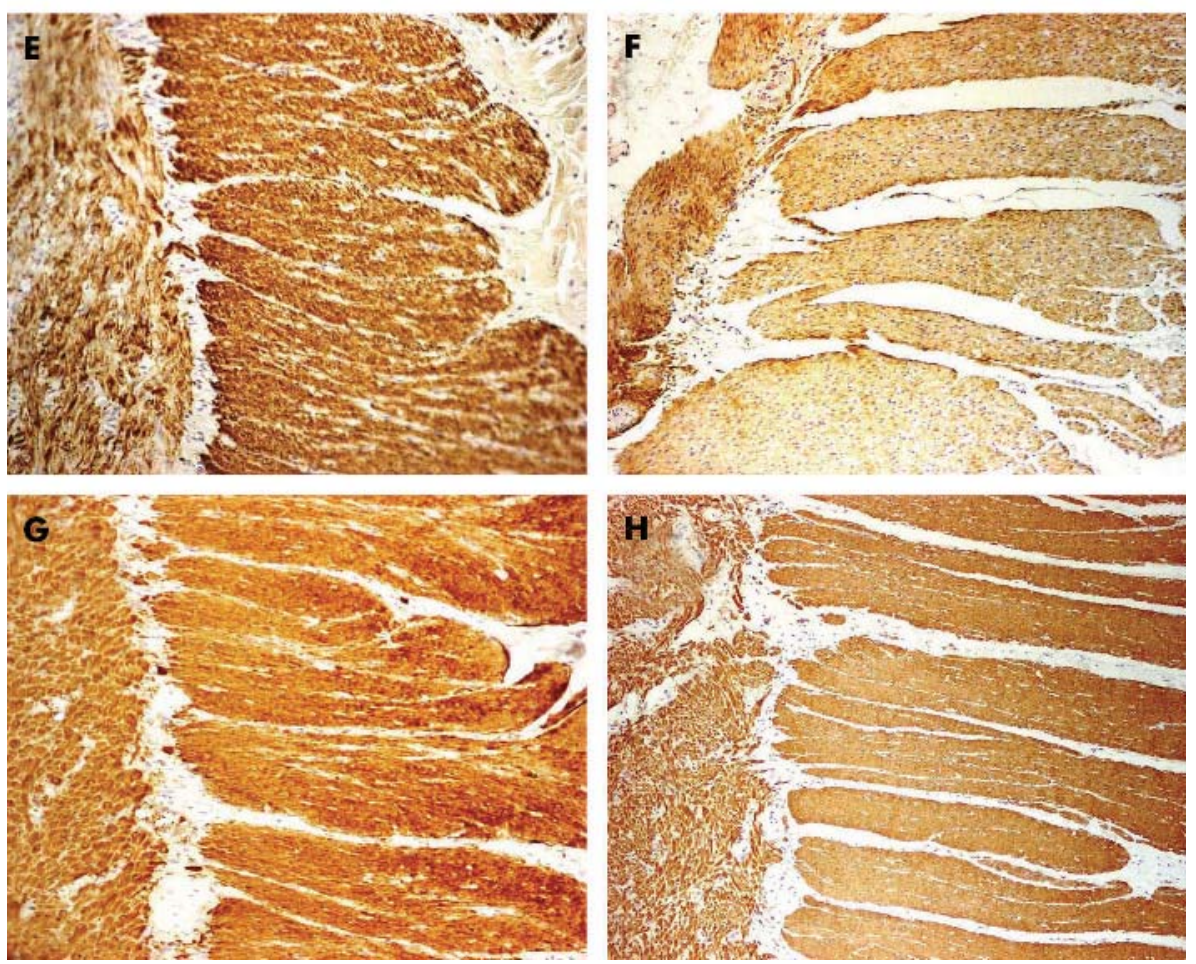

In all cases and controls, histological examination revealed morphologically normal submucosal and myenteric nerve plexuses and muscularis propria smooth muscle. In particular, the muscularis propria smooth muscle showed no evidence of muscle layer loss, inflammation, fibrosis, or inclusion bodies using haematoxylin and eosin and diastaseperiodic acid Schiff staining.

Formalin fixed and paraffin wax embedded sections of tissues were cut at $4 \mu \mathrm{m}$, dewaxed, and rehydrated in xylene and ethanol before antigen retrieval. Using the standard avidin-biotin-peroxidase complex staining technique, immunohistochemical staining was performed on all of the cases using the following primary antibodies: monoclonal anti-ASMA (clone 1A4; Sigma, Poole, Dorset, UK) at a 1/200 dilution, monoclonal anti-SMMHC (Dako, High Wycombe, UK) at a 1/200 dilution, and monoclonal anti-desmin (ICN Biomedicals, Basingstoke, UK) at a $1 / 30$ dilution. For each sample, an adjacent tissue section was included without primary antibody as a negative control.

Endogenous peroxidase was inhibited using $0.5 \%$ hydrogen peroxide in methanol. The sections were then rinsed in buffer at pH 7.6. For ASMA and SMMHC, antigen retrieval was 
Table 1 ASMA expression in cases and controls, as assessed by immunohistochemistry

\begin{tabular}{|c|c|c|c|c|c|}
\hline \multirow[b]{2}{*}{ Case } & \multirow{2}{*}{\multicolumn{2}{|c|}{ Age (years) Sex }} & \multirow[b]{2}{*}{ Sample } & \multicolumn{2}{|c|}{ ASMA expression } \\
\hline & & & & Circular & Longitudinal \\
\hline Case 1 & 41 & $\mathrm{~F}$ & Small & - & + \\
\hline Case 2 & 28 & $\mathrm{~F}$ & Small & - & + \\
\hline Case 3 & 54 & $\mathrm{~F}$ & Large & + & + \\
\hline \multirow[t]{2}{*}{ Control 1} & 83 & M & Small & - & + \\
\hline & & & Large & + & + \\
\hline \multirow{2}{*}{ Control 2} & 88 & $\mathrm{~F}$ & Small & - & + \\
\hline & & & Large & + & + \\
\hline \multirow{2}{*}{ Control 3} & 69 & $\mathrm{~F}$ & Small & - & + \\
\hline & & & Large & + & + \\
\hline \multirow{2}{*}{ Control 4} & 75 & M & Small & - & + \\
\hline & & & Large & + & + \\
\hline Control 5 & 85 & $\mathrm{~F}$ & Small & - & + \\
\hline Control 6 & 79 & M & Small & - & + \\
\hline Control 7 & 88 & $\mathrm{~F}$ & Small & - & + \\
\hline Control 8 & 78 & $\mathrm{~F}$ & Small & - & + \\
\hline \multirow{2}{*}{ Control 9} & 40 & $\mathrm{~F}$ & Small & - & + \\
\hline & & & Large & + & + \\
\hline \multirow[t]{2}{*}{ Control 10} & 50 & $\mathrm{~F}$ & Small & - & + \\
\hline & & & Large & + & + \\
\hline \multirow[t]{2}{*}{ Control 11} & 50 & M & Small & - & + \\
\hline & & & Large & + & + \\
\hline \multirow[t]{2}{*}{ Control 12} & 83 & $\mathrm{~F}$ & Small & W & + \\
\hline & & & Large & + & + \\
\hline \multirow[t]{2}{*}{ Control 13} & 72 & $\mathrm{~F}$ & Small & W & + \\
\hline & & & Large & + & + \\
\hline \multirow[t]{2}{*}{ Control 14} & 48 & M & Small & W & + \\
\hline & & & Large & + & + \\
\hline \multirow[t]{2}{*}{ Control 15} & 47 & $\mathrm{~F}$ & Small & W & + \\
\hline & & & Large & + & + \\
\hline
\end{tabular}

performed by placing the sections in a citrate buffer $(0.01 \mathrm{M}$ citric acid with $80 \mathrm{~g}$ of sodium hydroxide/litre, adjusted to pH 6.0). Sections to be stained for ASMA were heated in a microwave oven (Panasonic NN-5452, 800 Watts, seven minutes at high power and seven minutes at medium power) and those for SMMHC were pressure cooked (Tefal CLIP50 $\mathrm{X}$-PRESS at $13 \mathrm{lb}$ pressure for two minutes). Sections to be stained with desmin were not subjected to antigen retrieval but were treated with $1 \%$ bovine serum albumin for 30 minutes. The sections were incubated with the primary antibody: at room temperature for 30 minutes in the case of ASMA and desmin, and for 24 hours at $4^{\circ} \mathrm{C}$ for SMMHC. After rinsing the sections in buffer, immunoreactivity was demonstrated using the LSAB kit (Dako), followed by 3-3' diaminobenzidine-hydrogen peroxide solution. The slides were counterstained with haematoxylin, dehydrated, and mounted.

Each section was assessed by comparing the intensity of immunohistochemical labelling within the circular and longitudinal muscle layers of the muscularis propria, for each of the primary antibodies. Immunoreactivity within the muscularis mucosa and vessel walls acted as positive internal controls. Each section was scored independently by two consultant gastrointestinal pathologists (ACB and NJC).

\section{RESULTS}

Table 1 summarises the results. The small intestinal samples from cases 1 and 2 and from controls $1-11$ showed absent or minimal ASMA expression within the inner circular layer of the muscularis propria, with the exception of the innermost layer adjacent to the submucosa, where intense expression was present (fig 1A, B). Intense ASMA expression was present throughout the outer longitudinal muscle, muscularis mucosa, and vessel walls. In controls 12-15, small intestinal circular muscle ASMA expression was assessed as weak-there was slightly less intense expression within the inner circular muscle compared with the longitudinal muscle or the innermost circular muscle (fig $1 \mathrm{C}$ ). The large intestinal sample from patient 3 showed equally intense ASMA expression within the inner circular and outer longitudinal layers of the muscularis propria (fig 1D).

All cases and controls showed equally intense desmin and SMMHC expression within the inner circular and outer longitudinal muscle layers of the muscularis propria, muscularis mucosa, and vessel walls, within both the small and large intestine (fig $\mathrm{lE}-\mathrm{H}$ ).

\section{DISCUSSION}

We have demonstrated absent or minimal ASMA expression within the inner circular smooth muscle layer of the small intestinal muscularis propria in two cases of intestinal pseudo-obstruction and in most of our controls. This pattern of ASMA expression is the same as that described by Smith et al in a case of intestinal pseudo-obstruction. ${ }^{6}$ However, those authors did not find a lack of ASMA expression in control cases in which there was no history of pseudo-obstruction.

Our study and that of Smith et al found intense ASMA expression within the innermost circular muscle layer, even when expression in the remainder of the inner circular layer was absent. Smith et al suggested that this phenomenon might be related to the embryological development of the intestine being similar to that of rats, where the development of the innermost circular muscle layer appears as a distinct cell layer of actin positive cells in the perinatal period. ${ }^{7}$

\section{"Our study and that of Smith et al found intense $\alpha$ smooth muscle actin expression within the innermost circular muscle layer, even when expression in the remainder of the inner circular layer was absent"}

Our study and that of Smith et al used the same monoclonal antibody, specific for a single isoform of 


\section{Take home messages}

- Our findings do not support the hypothesis that smooth muscle $\alpha$ actin (ASMA) deficiency within the intestinal muscularis propria, as assessed by immunohistochemistry, is specifically associated with intestinal pseudoobstruction

- In fact, the absence of ASMA expression within the intestinal muscularis propria may be a normal finding

ASMA, to demonstrate the expression of ASMA. ${ }^{8}$ However, in our study, immunohistochemical staining was performed on formalin fixed and paraffin wax embedded tissue, whereas Smith et al used cryostat sections of snap frozen tissue. It is possible that this technical variation might account for the discrepancies between the findings in the control samples in the two studies.

Fromont-Hankard et al investigated immunohistochemical ASMA expression in large intestinal samples from patients with Hirschsprung's disease, using the same primary antiASMA antibody as in our study, on formalin fixed and paraffin wax embedded sections. ${ }^{9}$ Control material was sourced from morphologically normal large intestine and from dilated colons removed for intestinal pseudo-obstruction and obstructing colonic carcinoma. No differences in ASMA expression between the inner circular and outer longitudinal layers of the muscularis propria were found in aganglionic bowel segments or non-dilated controls. However, the dilated controls showed absent inner circular layer ASMA expression, except again for the innermost layer. This ASMA expression pattern was also seen in 30\% of normoganglionic tissue samples, without morphological changes in myocytes of the circular muscle and with faintly reduced or normal immunostaining with the antibody to pan-muscle actin. Therefore, Fromont-Hankard et al suggested that absent ASMA expression might be a secondary event related to chronic colonic obstruction and subsequent proximal dilatation. ${ }^{9}$ In our study, deficient ASMA expression was confined to the small intestine. We are not aware that the terminal ileum was significantly dilated in our control cases, although we cannot entirely exclude this as a possibility.

We conclude that there is insufficient evidence from our study to support the hypothesis that ASMA deficiency within the intestinal muscularis propria, as determined using immunohistochemistry on archival formalin fixed and paraffin wax embedded tissues, is specifically associated with clinical evidence of intestinal pseudo-obstruction. Our results suggest that the immunohistochemical demonstration of absent ASMA expression within the inner circular muscle layer of the intestinal muscularis propria, when performed on archival material, is not a reliable indicator of a primary pathological process occurring within the smooth muscle of the intestine, in cases of pseudo-obstruction. Indeed, we suggest that it may be a normal finding.

\section{Authors' affiliations}

E Gamba, N J Carr, A C Bateman, Department of Cellular Pathology, Southampton University Hospitals NHS Trust, Southampton S016 6YD, UK

\section{REFERENCES}

1 Martin JE, Smith W, Domizio P. Myopathies of the gastrointestinal tract. Recent Advances in Histopathology 1999;18:43-62.

2 Hartshorne DJ. Biochemistry of the contractile process in smooth muscle. In: Johnson LR, ed. Physiology of the gastrointestinal tract, I, 2nd ed. New York: Raven Press, 1987:423-82.

3 Fatigati V, Murphy RA. Actin and tropomyosin variants in the smooth muscles. $J$ Biol Chem 1984;259:1433-8.

4 Fitzgibbons PL, Chandrasoma PT. Familial visceral myopathy. Evidence of diffuse involvement of intestinal smooth muscle. Am J Surg Pathol 1987;11:846-54.

5 Christiensen J, Dent J, Malagelada JR, et al. Pseudo-obstruction. Gastroenterol Int 1990;3:107-19.

6 Smith VV, Lake BD, Kamm MA, et al. Intestinal pseudo-obstruction with deficient smooth muscle alpha-actin. Histopathology 1992;21:535-42.

7 Desa DJ. Alimentary tract. In: Wigglesworth JS, Singer DB, eds. Textbook of fetal and perinatal pathology, Vol. 2. Boston: Blackwell Scientific Publications, 1991:903-79.

8 Skalli O, Ropraz P, Trzeciak A, et al. A monoclonal antibody against alphasmooth muscle actin: a new probe for smooth muscle differentiation. J Cell Biol 1986; 103:2787-96

9 Fromont-Hankard G, Lafer D, Masood S. Altered expression of alpha-smooth muscle isoactin in Hirschsprung's disease. Arch Pathol Lab Med 1996; 120:270-4. 